\title{
Evaluation of C-Reactive Protein (CRP) in Early Diagnosis of Neonatal Septicemia
}

\author{
Ankita Nisarta ${ }^{1^{*}}$ and Rakesh Rajat $^{2}$ \\ ${ }^{1}$ Department of Microbiology, GMERS Medical College, Himmatnagar, Gujarat, India \\ ${ }^{2}$ Department of Microbiology, GMERS Medical College, Vadnagar, Gujarat, India \\ *Corresponding author
}

\section{Keywords}

CRP, Neonatal septicemia, Blood culture

Article Info

Accepted:

10 March 2019

Available Online:

10 April 2019

\section{A B S T R A C T}

Neonatal septicemia constitutes a significant cause of neonatal mortality \& morbidity in developing countries. It becomes essential to diagnose it as early as possible to reduce the mortality and treat with appropriate antibiotics. CRP is a sensitive \& good inflammatory marker. Definite diagnosis of septicemia depends on positive blood culture, which takes around 48-72 hrs. Result of CRP test is available within an hour in tertiary care centers and remote areas, where there is no availability of well equipped laboratory setup for blood culture. 315 Neonates with suspected sepsis were included. Under aseptic precautions, Blood samples were collected in blood culture bottle \& in Plain Vaccute. The CRP test was done by rapid slide latex agglutination method using the diagnostic kit (Tulip Diagnostics Ltd). Repeated Subcultures were performed. Identification of Organisms and Antibiotic sensitivity testing were done. Out of 315 cases, 180 (57\%) had positive CRP and $135(43 \%)$ cases had positive blood culture. The Most common organism isolated were Gram negative organisms (65\%) like Klebsiella 56(41\%) followed by Pseudomonas 19(14\%), E. coli 11(8\%), and Gram positive organisms (35\%) like CONS 39(29\%), Staphylococcus aureus 08(6\%) . Estimation of CRP is easy to perform, rapid. It can use as inflammatory marker in early diagnosis of neonatal septicemia which will help to prevent severe and life threatening complications.

\section{Introduction}

Neonatal septicemia is defined as a clinical syndrome characterized by systemic signs and symptoms due to generalized bacterial infection in the first four weeks of life $(1,5)$.

In developing countries, neonatal septicemia constitutes a significant cause of neonatal mortality \& morbidity.(2) Newborns of whole world especially those of third world countries are most vulnerable group for this illness. It is crucial to protect the newborns from infection as far as it is possible. (5)

Early diagnosis and proper treatment with appropriate antibiotics of neonatal septicemia can bring down the morbidity and mortality substantially. 


\section{Clinical manifestations}

Refusal to Feeds; Fever; Reduced Movement Poor Cry; Poor Weight Gain; Cyanosis ; Respiratory Distress; Hypothermia; Diarrhoea Vomiting; Abdominal Distension; Jaundice Convulsions $(2,3,6,17)$.

\section{Risk factors}

Prematurity

Very low birth weight

Exposure to invasive procedures

Frequent use of broad-spectrum antibiotics

Prolonged hospital stay

Premature rupture of membranes (PROM)

Premature onset of labour

Intrapartum maternal fever (6)

Fulminant and fatal course of infection may result from complications such as shock, disseminated intravascular coagulation and multi-system organ failure, mandating early diagnosis of this life-threatening condition for a timely treatment and a favorable outcome (1).

Definite diagnosis of septicemia depends on positive blood culture, which takes around 48$72 \mathrm{hrs}$.it is time consuming and demands a well equipped laboratory which is not available in most of the community hospitals (4). Hence, certain rapid diagnostic tests such as C-reactive protein, Micro-erythrocyte sedimentation rate, Buffy coat smear examination, Total WBC Count, Absolute Neutrophil count, Immature/Total Neutrophil count ratio and Platelet count collectively termed as the 'Sepsis Screen' is used, in addition to the Sepsis Score to diagnose.

Septicemia early and initiate a presumptive treatment while awaiting culture report (1). Therefore, the need is for a test that is cheap, easily performed with quick availability of reports. An ideal diagnostic test for neonatal sepsis should have maximum sensitivity and specificity (2). The knowledge of bacteriological profile and its antibiotic sensitivity patterns is of immense help in saving lives of neonates with septicemia. (7)

CRP is a sensitive, rapid \& very good inflammatory marker. Result of CRP test is available within an hour in community hospitals. CRP is synthesized within six to eight hours of exposure to an infective process or tissue damage. It has a half-life of 19 hours and may increase more than 1000fold during an acute phase response (2). Creactive protein (CRP) has been used as an acute phase reactant to diagnose and follow the course of infection in neonates. Its advantages include its very low serum levels in normal infants, a rapid rise within 12 to 24 hours of sepsis and a large incremental increase thereafter (4). C-reactive protein binds to several polysaccharides and peptide polysaccharides present in bacteria, fungi and parasites in the presence of calcium. These complexes activate the classical complement pathway, acting as opsonins and promoting phagocytosis. Together with complement components, CRP is the only acute phase protein directly involved in the clearance of microorganisms (2).

The profile of causative agents of neonatal septicemia varies in different geographical areas and from time to time. In India, Klebsiella pneumoniae is the most frequently isolated pathogen followed by Staphylococcus aureus. In developed nations, Group $B$ Streptococcus is implicated as the leading pathogen causing neonatal septicemia.

The recent challenge is in management of the morbid babies suffering from septicemia due to emergence of resistant strains to various classes of antibiotics and the changing pattern of susceptibility from time to time. It is essential to know the changing pattern of 
antibiotic sensitivity of predominant organisms to plan a strategy for treatment at a particular geographical area (6).

The main objective of this study to diagnose septicemia early using C-reactive protein (CRP) test to prevent severe and life threatening complications \& to isolate \& identify the organism responsible for neonatal septicemia and their antibiotic sensitivity patterns.

\section{Materials and Methods}

The study was conducted at Microbiology Laboratory, Sir T. Hospital, Bhavnagar from June-2013 to November-2013.

315 Neonates with suspected sepsis with the age of first 28 days of life were included in study.Blood culture was the gold standard in this study. Under aseptic precautions, Blood samples were collected in to bottle of $10 \mathrm{ml}$ brain heart infusion broth \& in Plain Vaccute.

The CRP test was done by rapid slide latex agglutination method using the diagnostic kit supplied commercially by Tulip Diagnostics Ltd, which detects serum levels greater than
$0.6 \mathrm{mg} / \mathrm{dl}$ of CRP.

Blood culture bottles were incubated for 7 days. Repeated Subcultures were performed on Blood agar, Mac Conkey's agar and Chocolate agar very alternate day till the 7th day, for possibility of any growth.

In cases where no growth was obtained after 7 days of incubation, then it was considered as a negative blood culture. If growth is there, than Isolates were identified by colony morphology, gram stain, and biochemical tests. Antibiotic sensitivity testing was done by Kirby Bauer disk diffusion method.

\section{Results and Discussion}

Out of 315 samples, 179 (57\%) showed positive CRP and $136(43 \%)$ cases showed positive blood culture.

Gram negative organisms formed the majority of the isolates as compared to Gram positive organisms (65\% vs. $35 \%$ respectively).

Overall sensitivity of CRP is $57 \%$ (Table 1 and 2).

Table.1 Statistical analysis of CRP test

\begin{tabular}{|l|l|}
\hline Validity of CRP level Values & In present study \\
\hline Sensitivity & $87 \%$ \\
\hline Specificity & $71 \%$ \\
\hline Positive predictive Value & $74 \%$ \\
\hline Negative Predictive value & $86 \%$ \\
\hline
\end{tabular}


Table.2 Comparison of results of blood culture and CRP test

\begin{tabular}{|l|l|l|l|l|l|l|} 
& $\begin{array}{l}\text { Total culture } \\
\text { positive }\end{array}$ & $\begin{array}{l}\text { Total CRP } \\
\text { positive }\end{array}$ & $\begin{array}{l}\text { Culture } \\
\text { positive } \\
\text { CRP } \\
\text { positive }\end{array}$ & $\begin{array}{l}\text { Culture } \\
\text { positive } \\
\text { CRP } \\
\text { negative }\end{array}$ & $\begin{array}{l}\text { Culture } \\
\text { Negative } \\
\text { CRP } \\
\text { positive }\end{array}$ & $\begin{array}{l}\text { Culture } \\
\text { Negative } \\
\text { CRP } \\
\text { Negative }\end{array}$ \\
\hline No. of cases & 136 & 179 & 132 & 19 & 47 & 117 \\
\hline Percentage & $43 \%$ & $57 \%$ & $42 \%$ & $6 \%$ & $15 \%$ & $37.5 \%$ \\
\hline
\end{tabular}

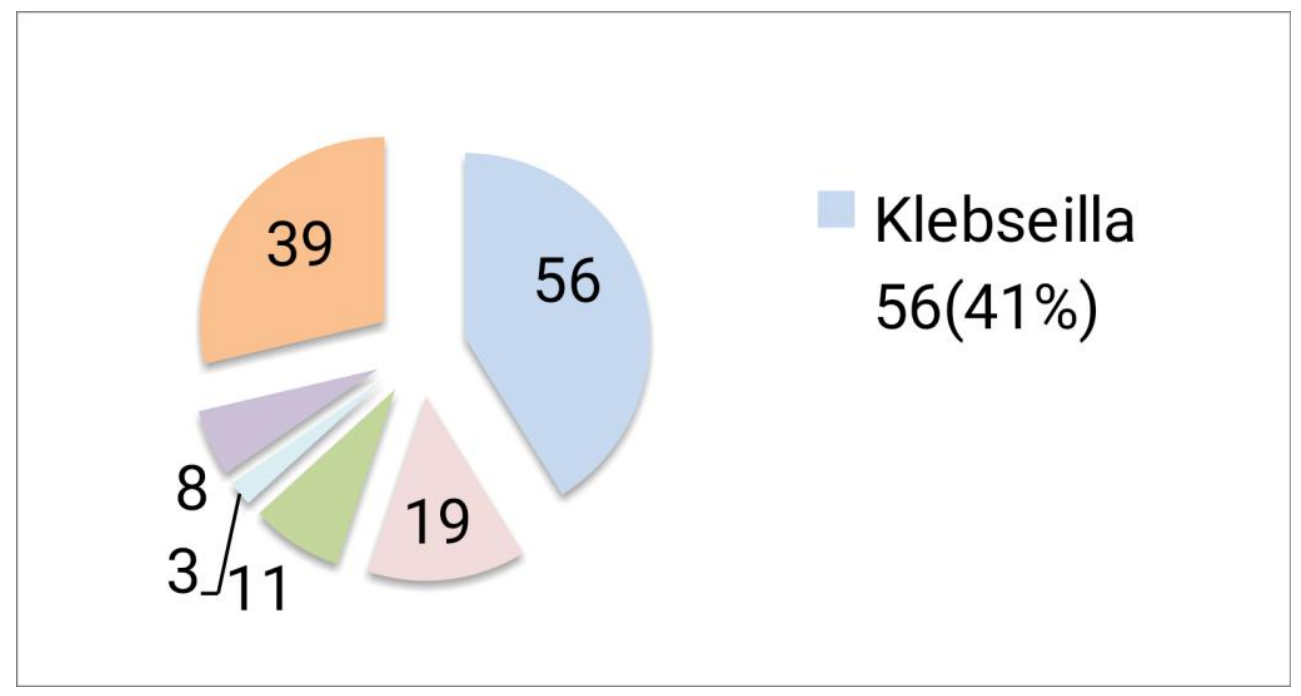

In this present study, of the 315 clinically suspected septicemia cases, $136(43 \%)$ were culture positive and $179(57 \%)$ were culture negative. The ratio of culture positive cases was higher among males than the females in the present study. The male preponderance in neonatal septicemia may be linked to the $\mathrm{X}$ linked immunoregulatory gene factor contributing to the host's susceptibility to infections in males.(1) There may be another two reasons. First, male sex carries a higher risk for sepsis as compared to females. Second, parents of our country are more concerned for their boys and bring them earlier to the hospital. (3)

In the present study, 136/315 cases studied were culture positive, giving a positivity rate of $43 \%$. The low culture positivity in these studies may be due to intrapartum administration of antibiotics to mothers which can affect the blood culture results in neonate, Various factors like administration of antibiotics to mother or baby before blood collection, possibility of infection with fastidious organisms and anaerobes, etc. pose difficulty in isolation unless advanced automated or molecular techniques are employed, which may be expensive in routine laboratory protocol $(1,6,10)$.

Gram negative organisms formed the majority of the isolates as compared to Gram positive organisms (65\% vs $35 \%$ respectively) in the present study. Klebsiella pneumoniae $(41 \%)$ was the predominant isolate, which correlates with study of Twinkle N Gandhi et al., (2, 13). The causative organisms of neonatal 
sepsis vary with time and place. There is increasing trend of antibiotic resistance to the commonly used and available drugs. Continuous surveillance is needed to monitor changing epidemiology of pathogens and antibiotic susceptibility pattern (16).

Amongst the gram positive organisms, we isolated $29 \% \quad(39 / 136)$ CONS which corresponds to $6 \%(8 / 136)$ S.aureus. An incidence of $6.92 \%(9 / 130)$ for CONS observed in study of Twinkle N Gandhi et al., (2) found to be a matter of concern because this bacterium is often regarded as a contaminant, possibly from the skin, (2) but I Roy et al., (8) opined that the presence of this bacterium in the blood can no longer be taken as contamination especially in patients in critical care units (8).

The predominant gram negative isolates of Klebsiella pneumoniae and E. coli showed a pattern of multi drug resistance, which is consistent with observations cited by Jayanta Debnath et al., This indicates the emergence of highly resistant mutants especially found with nosocomial pathogens. However, all of these isolates were sensitive to Imipenem, followed by Piperacillin-tazobactum. And Amikacin with significant resistance to Fluoroquinolones and Penicillins (6).

Staphylococcus aureus showed $100 \%$ sensitivity to Vancomycin and resistance to Ampicillin and penicillin in all isolates. They were significantly sensitive to Cephalosporins like Cephalexin, Ceftriaxone and Cefotaxime as also observed in other reference studies. The isolate was 93\% sensitive to Amikacin compared to $32 \%$ for Gentamicin.(6)

There is no single reliable test for the early definite diagnosis of neonatal sepsis, and therefore, there is a continuing search for a new infection marker. The $\mathrm{C}$-reactive protein has been the most analyzed parameter for the detection of bacterial infections for years (Monneret et al., 2008; Cheisa et al., 1998).

The present study was undertaken to assess the utility of CRP test as marker for diagnosis of neonatal septicemia. In our study, we found sensitivity of CRP $87 \%$, specificity $71 \%$, positive predictive value (PPV) $74 \%$ \& negative predictive value (NPV) $86 \%$ considering blood culture as gold standard method.Our results are comparable with the study done by Anwar Zeb1Manisha Yadav, which shows that C-reactive protein had higher sensitivity $94.01 \%$ and $88 \%$ for proven and probable sepsis and specificity $6.71 \%$ and $84 \%$ for proven sepsis in early and late onset episodes (11). The lower scores for positive predictive values reflect the lag period between the onset of infection and production of sufficient CRP to exceed the normal range $(12,15)$.

Piperacillin/tazobactum and cefoperazone/ sulbactum were the two most effective antibiotics against Gram-negative organisms.

The bacteriological profile of septicaemia keeps changing with the passage of time from region to region and hospital to hospital, in the same city or country. The emergence of resistant bacteria in NICU settings leads to failure in the treatment of neonatal septicaemia (7).

Effat Hisamuddin et al., stated that monitoring of CRP overtime may be used to in determining the response of the treatment after the primary diagnosis. They were discharged home after 5 days of intravenous antibiotic therapy. Effat Hisamuddin et al., concluded in their study that quantitative CRP is arapid, sensitive diagnostic marker for identification of sepsis in preterm infants .In our study, we also found CRP a good indicator of neonatal sepsis as qualitative 
status of CRP helped in identification of neonatal sepsis and also in deciding the line of management of the patient (9).

Serial measurements of serum CRP levels are useful in monitoring the course of neonatal septicaemia. It provides an early indication of response of treatment. It can help in decision of initiating or discontinuing antibiotic therapy. The persistence or insignificant decline of serum CRP with treatment signifies about inadequate treatment or development of complications (14).

The marked difference of result among studies evaluating C-reactive protein as useful marker can be explained by non-availability of universally acceptable definition of neonatal sepsis, difference in reference range values and environmental influence on the results in different setups. The test for estimation of CRP is easy to perform and results will be available in minutes. Furthermore, it can also be used effectively in neonates who had already used antibiotics (18).

The antimicrobial sensitivity pattern differs in different studies as well as at different times. This is because of emergence of resistant strains as a result of indiscriminate use of antibiotics.

In conclusion, CRP can be used as a tool in early diagnosis of Neonatal Septicemia specially where there is lack of investigational facilities.

Advantages of CRP include its very low serum levels in normal infants, a rapid rise within 12 to 24 hours of sepsis and a large incremental increase thereafter. Neonatal septicemia is a life threatening emergency, and rapid treatment with antibiotics is essential for a favorable outcome. Classical empirical treatment of neonatal sepsis consists of amoxicillin \& an amino glycoside.

\section{References}

1. Rekha Sriram et al., Correlation of Blood culture results with the Sepsis score and the Sepsis screen in the diagnosis of Neonatal Septicaemia, Int J Biol Med Res. 2011; 2(1): 360-368.

2. Chauhan $\mathrm{S}$ et al., C-reactive Protein (Crp) In early diagnosis of neonatal septicemia, national Journal Of Medical Research, Volume 2|Issue 3|July Sept 2012.

3. Twinkle N Gandhi1, Manish G Patel2, Mannu R Jain et al., Utility Of $\mathrm{C}$ Reactive Protein As Inflammatory Marker In Early Diagnosis Of Neonatal Septicaemia: a Cross Sectional Study, national Journal Of Medical Research, 2(4): 2012.

4. S Afroza, F Begumb et al., Co-relation between Sepsis Score and Blood Culture Report in Neonatal Septicaemia, Journal of Bangladesh College of Physicians and Surgeons, Vol. 26, No. 2, May 2008.

5. Parviz Ayazi, Mohammad Mahdi Daneshi et al., The Role of Serial Serum C-Reactive Protein Level in the Diagnosis of Neonatal Infection., Hassan, Iranian Journal of Pediatrics Society, Volume 1, Number 1, 2007: 4751.

6. M M. Khassawneh, W. A. Hayajnehet al., Diagnostic Markers for Neonatal Sepsis: Comparing C-reactive Protein, Interleukin-6 and Immunoglobulin, The Authors Journal compilation 2007 Blackwell Publishing Ltd. Scandinavian Journal of Immunology 65, 171-175.

7. Ho LY et al., Sepsis in young infants Rational approach to early diagnosis and treatment. Singapore, Med J 1992; 33:119-22.

8. I Roy, A Jain, M Kumar et al., Bacteriology Of Neonatal Septicaemia In 
A TertiaryCare Hospital Of Northern India, Indian Journal Of Medical Microbiology, (2002) 20 (3):156-159.

9. Hisamuddin E, Hisam A et al., Validity of C-reactive protein (CRP) for diagnosis of neonatal sepsis. Pak J Med Sci2015; 31(3):527-531.

10. Raina Dimple, Rana Jyoti et al., Prevalence of Gram negative bacteria causing neonatal septicemia in a tertiary care hospital of Dehradun, Uttarakhand, India, Int.J.Curr.Microbiol.App.Sci (2016) 5(1): 136-147.

11. Jan AZ, Zahid SB, Ahmad S. et al., Role of $\mathrm{C}$-reactive protein in diagnosing neonatal sepsis. Khyber Med Univ J 2012; 4(4): 161-164.

12. Kumar, R., Musoke, R., Macharia, W.et al., Validation of c-reactive protein in the early diagnosis of neonatal sepsis in a tertiary care hospital in Kenya.. East African Medical Journal, 87(6), 255-261.

13. Gunjan Mehrotra et al., Study of C reactive protein in neonatal sepsis, Int $\mathbf{J}$ Contemp Pediatr. 2017 May; 4(3): 890895.
14. Swati M. Bapat et al., C-Reactive Proteins as diagnostic and prognostic indicator in neonatal sepsis, IJBAR (2018) 09 (03), 117-120.

15. Manisha Yadav et al., Role of C-reactive protein in early diagnosis of neonatal sepsis, International Journal of Current Research Vol. 7, Issue, 07, pp.1828018282, July, 2015.

16. Lakhey A et al., Role of sepsis screening in early diagnosis of neonatal sepsis, Journal of Pathology of Nepal (2017) Vol. 7, 1103 -1110.

17. Santhakumar Sundarapandian et al., Serial Serum C-reactive Protein in the Diagnosis of Neonatal Sepsis: A Crosssectional Study, Indian Journal of Neonatal Medicine and Research. 2017 Apr, Vol-5(2): PO10-PO15.

18. D. Jeyaganguli et al., Diagnostic Value of C - Reactive Protein and Hematological Markers in Neonatal Sepsis, Int.J.Curr.Microbiol.App.Sci (2018) 7(3): 722-727.

\section{How to cite this article:}

Ankita Nisarta and Rakesh Rajat. 2019. Evaluation of C-Reactive Protein (CRP) in Early Diagnosis of Neonatal Septicemia. Int.J.Curr.Microbiol.App.Sci. 8(04): 1276-1282. doi: https://doi.org/10.20546/ijcmas.2019.804.146 